\title{
The Factor Domains that Result from Uppers to Prime Ideals in Polynomial Rings
}

\section{DAVID EARL DOBBS}

Department of Mathematics, University of Tennessee, Knoxville, Tennessee 37996-

0612

e-mail : dobbs@math.utk.edu

ABSTRACT. Let $P$ be a prime ideal of a commutative unital ring $R ; X$ an indeterminate; $D:=R / P ; L$ the quotient field of $D ; F$ an algebraic closure of $L ; \alpha \in L[X]$ a monic irreducible polynomial; $\xi$ any root of $\alpha$ in $F$; and $Q=\langle P, \alpha\rangle$, the upper to $P$ with respect to $\alpha$. Then $R[X] / Q$ is $R$-algebra isomorphic to $D[\xi]$; and is $R$-isomorphic to an overring of $D$ if and only if $\operatorname{deg}(\alpha)=1$.

\section{Introduction}

All rings and algebras considered in this note are commutative with $1 \neq 0$; all subrings/subalgebras and algebra homomorphisms are unital; and $X$ denotes an indeterminate over the ambient coefficient ring(s). Our main concern here is the notion of an upper, which was implicit in a brief passage [5, page 25] introducing the basic facts about the Krull dimension of a polynomial ring $R[X]$; made explicit, with suggestive and helpful notation, in case $R$ is a (commutative integral) domain, in [6, pages 706-708]; and generalized to the case of an arbitrary coefficient ring $R$ in [3, pages 291-292]. The definition of an "upper to $P$ " depends on the following data. Let $P$ be a prime ideal of a commutative unital ring $R ; X$ an indeterminate; $D:=R / P ; L$ the quotient field of $D$; and $\alpha \in L[X]$ a monic irreducible polynomial. Then the upper to $P$ with respect to $\alpha$ is defined to be $\langle P, \alpha\rangle:=\{h \in R[X] \mid$ the canonical image of $h$ in $D[X]$ is divisible by $\alpha$ in $L[X]\}$. As the passages cited above show, the "upper" concept is important because, if $P$ is a prime ideal of a $\operatorname{ring} R$, the prime ideals $Q$ of the polynomial $\operatorname{ring} R[X]$ such that $Q \cap R=P$ are of two kinds: either $Q=P^{*}:=P R[X]$ or $Q=\langle P, \alpha\rangle$ for some monic irreducible polynomial $\alpha \in L[X]$. Since it is easy to see that $R[X] / P^{*}$ is $R$-algebra isomorphic to $(R / P)[X]$, the question arises as to the nature of the factor domains of the form $R[X] /\langle P, \alpha\rangle$. We answer this question in Theorem 2.2 (a) below. The answer is elegant and its proof is elementary. Using the above notation, we show in Theorem 2.2 that $R[X] /\langle P, \alpha\rangle$ is $R$-algebra isomorphic to $D[\xi]$, where $\xi$ denotes any given

Received September 12, 2009; accepted January 27, 2010.

2000 Mathematics Subject Classification:Primary 13A15; Secondary 13G05, 13B25, $13 B 30$.

Key words and phrases: Commutative ring, prime ideal, polynomial ring, upper, integral domain, factor ring, degree. 
root of $\alpha$ in an algebraic closure of $L$. In this way, we see that factor domains with respect to uppers give another way of describing a class of domains that has been the subject of considerable attention in a number of classical contexts (cf. [7, Theorem], [8, Proposition 3.11], [1, Theorem]).

\section{Results}

Our first result explains how our basic question reduces to working with coefficient rings that are domains and uppers to 0.

Lemma 2.1. Let $P$ be a prime ideal of a ring $R ; D:=R / P ; L$ the quotient field of $D$; and $\alpha \in L[X]$ a monic irreducible polynomial. Then $R[X] /\langle P, \alpha\rangle$ is $R$-algebra isomorphic to $D[X] /\langle 0, \alpha\rangle$.

Proof. The canonical projection $R \rightarrow R / P$ extends to a surjective $R$-algebra homomorphism $h: R[X] \rightarrow D[X]$ that send $X$ to $X$; thus, $h\left(\sum_{i=0}^{n} r_{i} X^{i}\right)=\sum_{i=0}^{n}\left(r_{i}+\right.$ $P) X^{i}$ for any polynomial $\sum_{i=0}^{n} r_{i} X^{i} \in R[X]$. Composing $h$ with a canonical projection, we obtain a surjective $R$-algebra homomorphism $g: R[X] \rightarrow D[X] /\langle 0, \alpha\rangle$, satisfying $g\left(\sum_{i=0}^{n} r_{i} X^{i}\right)=\sum_{i=0}^{n}\left(r_{i}+P\right) X^{i}+\langle 0, \alpha\rangle$. Clearly, $\operatorname{ker}(g)=\langle P, \alpha\rangle$, and so the assertion follows from the First Isomorphism Theorem for $R$-algebras.

We next present our main result. Recall that if $D$ is a domain with quotient field $L$, then an overring of $D$ is any $D$-subalgebra of $L$ (that is, any subring of $L$ that contains $D)$.

Theorem 2.2. Let $P$ be a prime ideal of a ring $R ; D:=R / P ; L$ the quotient field of $D ; F$ an algebraic closure of $L$; and $\alpha \in L[X]$ a monic irreducible polynomial. Then:

(a) $R[X] /\langle P, \alpha\rangle$ is $R$-algebra isomorphic to $D[\xi]$ for each root $\xi$ of $\alpha$ in $F$.

(b) $R[X] /\langle P, \alpha\rangle$ is $R$-algebra isomorphic to an overring of $D$ if and only if $\operatorname{deg}(\alpha)=1$.

Proof. By Lemma 2.1, we can replace $R$ with $D$ and also replace $P$ with 0 . In other words, we can assume, without loss of generality, that $R$ is a domain and $P=0$.

(a) As in the proof of Lemma 2.1, we obtain an explicit surjective $R$-algebra homomorphism $h: R[X] \rightarrow D[X], \sum_{i=0}^{n} r_{i} X^{i} \mapsto \sum_{i=0}^{n}\left(r_{i}+P\right) X^{i}$. Note that $\operatorname{ker}(h)=P R[X]=: P^{*} \subseteq\langle P, \alpha\rangle$. Moreover, $h$ carries the set $\langle P, \alpha\rangle$ onto the set $S:=\{g \in D[X]|\alpha| g$ in $L[X]\}$. (In fact, $S$ is a prime ideal of $D[X]$.) It then follows from a standard homomorphism theorem that $R[X] /\langle P, \alpha\rangle$ and $D[X] / S$ are isomorphic as $R$-algebras. Therefore, it suffices to prove that $D[X] / S \cong D[\xi]$ as $R$-algebras (where $\xi$ denotes any given root of $\alpha$ in $F$ ).

To simplify matters, let us use the above reduction, so that $R$ is a domain and $P$ is the prime ideal 0 of $R$. Our task is to show that $R[X] / S \cong R[\xi]$ as $R$-algebras. But since $\alpha$ is the minimum polynomial of $\xi$ over $L$, it follows that $S=R[X] \cap \alpha L[X]$ is the kernel of the surjective $R$-algebra (evaluation) homomorphism $e: R[X] \rightarrow R[\xi]$ that sends $X$ to $\xi$. Hence, the required isomorphism follows by applying the First Isomorphism Theorem for $R$-algebras to $e$. 
(b) Choose a root $\xi$ of $\alpha$ in $F$. Recall that we have reduced to the case $R=D$ and $P=0$. Thus, $R[X] /\langle P, \alpha\rangle$ is $R$-algebra isomorphic to an overring of $D$ if and only if there is an injective $R$-algebra homomorphism $R[X] /\langle 0, \alpha\rangle \rightarrow L$; that is, by (a), if and only if there is an injective $R$-algebra homomorphism $g: R[\xi] \rightarrow L$.

Assume first that such $g$ exists. We will show that $\operatorname{deg}(\alpha)=1$. Taking a common denominator for the coefficients of $\alpha$, we can write $\alpha=\beta / r$ for some $\beta \in R[X]$ and some nonzero element $r \in R$. Consequently, $\beta(\xi)=r \alpha(\xi)=r \cdot 0=0$. Also, since $g$ is an $R$-algebra homomorphism, we see that $\beta(g(\xi))=g(\beta(\xi))$. Thus, $\eta:=g(\xi) \in L$ satisfies

$$
\alpha(\eta)=\alpha(g(\xi))=\frac{1}{r} \beta(g(\xi))=\frac{1}{r} g(\beta(\xi))=\frac{1}{r} g(0)=\frac{1}{r} \cdot 0=0 ;
$$

that is, $\eta$ is a root of $\alpha$ in $L$. Since $\alpha$ is irreducible in $L[X]$, it follows that $\operatorname{deg}(\alpha)=1$.

Conversely, suppose that $\operatorname{deg}(\alpha)=1$. Then $\alpha=X-\delta$ for some $\delta \in L$. Note that $\delta$ is a root of $\alpha$. Therefore, by (a), $R[X] /\langle P, \alpha\rangle$ is $R$-algebra isomorphic to $R[\delta]$, which is an overring of $R$ (that is, of $D$ ).

The following is a useful restatement of Theorem 2.2 (a).

Corollary 2.3. Let $P$ be a prime ideal of a ring $R ; D:=R / P ; L$ the quotient field of $D$; and $F$ an algebraic closure of $L$. Then, up to $R$-algebra isomorphism, the rings of the form $R[X] / Q$, where $Q$ ranges over the set of uppers to $P$, are the same as the rings of the form $D[\xi]$, where $\xi$ ranges over (the set of elements of ) $F$.

Proof. In view of Theorem 2.2 (a), it remains only to show that if $\xi \in F$, then there exists some $\alpha$, a monic irreducible polynomial in $L[X]$, such that $D[\xi]$ is $R$-algebra isomorphic to $R[X] /\langle P, \alpha\rangle$. Choose $\alpha \in L[X]$ to be the minimum polynomial of $\xi$ over $L$. Then an application of Theorem 2.2 (a) completes the proof.

It is known that if $P$ is a prime ideal of a $\operatorname{ring} R$ and $\alpha, \beta$ are distinct monic polynomials that are each irreducible over the quotient field of $R / P$, then $\langle P, \alpha\rangle$ and $\langle P, \beta\rangle$ are unequal and, in fact, incomparable under inclusion (cf. [3, Lemma $2.1(\mathrm{a})]$ ). In view of Corollary 2.3, this raises the following question. If (using the above notation) $\xi$ and $\eta$ are elements of an algebraic closure of the quotient field of $R / P$ such that $D[\xi]$ and $D[\eta]$ are isomorphic as $R$-algebras, must it be the case that $D[\xi]=D[\eta]$ ? We will answer this question in Remark 2.4 (a) and a related question in Remark 2.4 (b).

Remark 2.4. (a) We proceed to answer the above question, assuming for simplicity that $R$ is a domain and $P=0$. Let $R$ be a domain with quotient field $L$, let $F$ be an algebraic closure of $L$, and let $\xi$ and $\eta$ be elements of $F$ such that $R[\xi]$ and $R[\eta]$ are isomorphic as $R$-algebras. Then, if $\xi$ and $\eta$ are each elements of $L$, then $R[\xi]=R[\eta]$. However, if at least one of $\xi, \eta$ does not belong to $F$, then it need not be the case that $R[\xi]=R[\eta]$.

To prove the first assertion, assume that $\xi$ and $\eta$ are each elements of $L$. Then $R[\xi]$ and $R[\eta]$ are $R$-algebra isomorphic overrings of $R$ (that are inside the same 
quotient field of $R$ ). Under these conditions, it is known (see the first paragraph of [4, Remark $2.8(\mathrm{a})]$ ) that these overrings must coincide.

Finally, we will give an example where $R[\xi]$ and $R[\eta]$ are distinct but $R$-algebra isomorphic $R$-subalgebras of $F$. Take $R:=\mathbb{Z}, P:=0$, and view $F \subseteq \mathbb{C}$. Choose $a, b \in \mathbb{Q}(=L)$ with $2 a \notin \mathbb{Z}$ and $b \neq 0$. Let $\xi:=a+b i$ and $\eta:=a-b i$ (where, as usual, $i:=\sqrt{-1} \in \mathbb{C}$ ). It is easy to check that $\xi$ and $\eta$ have the same minimum polynomial over $L$, namely, $\alpha:=X^{2}-2 a X+a^{2}+b^{2}$. Therefore, by Theorem 2.2 (a), $R[\xi]$ and $R[\eta]$ are each $R$-algebra isomorphic to $R[X] /\langle P, \alpha\rangle$ (and, hence, $R$-algebra isomorphic to each other). However, $R[\xi] \neq R[\eta]$, since the condition $2 a \notin \mathbb{Z}$ ensures that

$$
\eta=a-b i=2 a+(-1)(a+b i)=2 a+(-1) \xi \in(\mathbb{Q}+\mathbb{Q} \xi) \backslash(\mathbb{Z}+\mathbb{Z} \xi)=L[\xi] \backslash R[\xi]
$$

(b) We next answer another question that is raised by Corollary 2.3 (and also motivated by (a)). With $P$ a prime ideal of a ring $R$ and $\alpha, \beta$ monic irreducible polynomials over the quotient field of $R / P$ such that $R[X] /\langle P, \alpha\rangle \cong R[X] /\langle P, \beta\rangle$ as $R$-algebras, can it be the case that $\alpha \neq \beta$ ? In view of the above results, we can rephrase this question as follows. If $R$ is a domain with quotient field $L$ and $\alpha, \beta$ are distinct monic irreducible polynomials over $L$, can it be the case that $R[\xi]$ and $R[\eta]$ are isomorphic $R$-algebras, where $\xi$ and $\eta$ are roots of $\alpha$ and $\beta$, respectively, in a given algebraic closure of $L$ ?

The answer is in the affirmative. We illustrate this next in the most trivial case possible, namely, where $R$ is an algebraically closed field (so that, using the earlier notation, $R=D=L=F$ and, of course, $P=0$ ). Under this assumption, choose distinct elements $\lambda, \mu \in R$; and put $\alpha:=X-\lambda, \beta:=X-\mu \in R[X]$. Take $\xi$ and $\eta$ to be roots of $\alpha$ and $\beta$, respectively, in $R$; that is, $\xi=\lambda$ and $\eta=\mu$. Of course, $R[\xi]=R$ is $R$-algebra isomorphic to $R=R[\eta]$, although $\alpha \neq \beta$.

(c) We next pursue the idea from (b) of considering the degenerate case where the coefficient ring $R$ is a field. This brings to mind a classic result often referred to as the theorem of Cauchy, Kronecker and Steinitz. This result gives the usual construction of a field extension $K$ of a given field $k$ such that $K$ contains a root of a given irreducible polynomial $f \in k[X]$. Related analysis shows that if $\xi, \eta$ are each roots of $f$ in $K$, then the fields $k[\xi]$ and $k[\eta]$ are isomorphic $k$-algebras (since each is $k$-algebra isomorphic to $k[X] / f k[X])$. Note that Theorem 2.2 (a) implies a generalization of this classical fact, in that the coefficient ring can be an arbitrary domain. Indeed, Theorem 2.2 (a) shows that if $D$ is a domain with quotient field $L$ and $f \in L[X]$ is a monic irreducible polynomial with roots $\xi, \eta, \ldots$ in some algebraic closure of $L$, then $D[\xi] \cong D[\eta]$ as $D$-algebras (since each is $D$-algebra isomorphic to $D[X] /\langle 0, f\rangle)$.

(d) Some special cases (or variants thereof) of Theorem 2.2 (a) have appeared in the literature. For instance, [1, Lemma 1] states (when paraphrased using the above notation) that if $R$ is an integrally closed domain with quotient field $L$ and the element $\xi$ is integral over $R$, then $R[X] / \alpha R[X] \cong R[\xi]$ as $R$-algebras, where $\alpha \in R[X]$ denotes the minimum polynomial of $\xi$ over $L$. This is a special case of 
Theorem 2.2 (a) since the extra assumptions on $R$ and $\xi$ in [1, Lemma 1] ensure that $\alpha R[X]=\alpha L[X] \cap R[X]=\langle 0, \alpha\rangle$. Of course, the domains $R$ that we considered above need not be integrally closed; the algebraic elements $\xi$ that we considered above need not be integral; and so the monic irreducible polynomials $\alpha \in L[X]$ that we considered need not have all their coefficients in $R$. One should note that [1, Lemma 1] is slightly more general that suggested above, in that it permits the integral element $\xi$ to be taken from an arbitrary $L$-algebra (which is not necessarily contained in an algebraic closure of $L$ ).

Finally, we note that [2, Lemma 4.4] can be viewed as a weak version of Theorem 2.2 (a). Indeed, using the notation of Theorem 2.2 (a), we can paraphrase [2, Lemma 4.4] to say that if $R$ is a domain and $\langle P, \alpha\rangle$ is an upper to some prime ideal $P$ of $R$, then the domain $R[X] /\langle P, \alpha\rangle$ is algebraic over $D:=R / P$. However, we believe that the stronger conclusions in Theorem 2.2 (a) and Corollary 2.3 have not appeared earlier in the literature.

\section{References}

[1] T. Albu, On a paper of Uchida concerning simple finite extensions of Dedekind domains, Osaka J. Math., 16(1979), 65-69.

[2] A. Bouvier, D. E. Dobbs and M. Fontana, Universally catenarian integral domains, Adv. Math., 72(1988), 211-238.

[3] D. E. Dobbs and M. Fontana, Universally incomparable ring-homomorphisms, Bull. Austral. Math. Soc., 29(1984), 289-302.

[4] D. E. Dobbs and J. Shapiro, A classification of the minimal ring extensions of an integral domain, J. Algebra, 305(2006), 185-193.

[5] I. Kaplansky, Commutative Rings, rev. ed., Univ. Chicago Press, Chicago, 1974.

[6] S. McAdam, Going down in polynomial rings, Can. J. Math., 23(1971), 704-711.

[7] K. Uchida, When is $\mathbb{Z}[\alpha]$ the ring of integers?, Osaka J. Math., 14(1977), 155-157.

[8] H. Uda, Incomparability in ring extensions, Hiroshima Math. J., 9(1979), 451-463. 